\title{
Adaptive Heuristics That (Could) Fit: Information Search and Communication Patterns in an Online Forum of Investors Under Market Uncertainty
}

\author{
Niccolo Casnici \\ University of Brescia \\ Marco Castellani \\ University of Brescia \\ Flaminio Squazzoni \\ University of Brescia
}

See next page for additional authors

Follow this and additional works at: https://arrow.tudublin.ie/scschcomart

Part of the Computer Sciences Commons

\section{Recommended Citation}

Casnici, N., Castellani, M., Squazzoni, F., Testa, M., \& Dondio, P. (2019). Adaptive Heuristics That (Could) Fit: Information Search and Communication Patterns in an Online Forum of Investors Under Market Uncertainty. Social Science Computer Review, 37(6), 734-749. DOI: 10.1177/0894439318794412

This Article is brought to you for free and open access by the School of Computer Sciences at ARROW@TU Dublin. It has been accepted for inclusion in Articles by an authorized administrator of ARROW@TU Dublin. For more information, please contact arrow.admin@tudublin.ie, aisling.coyne@tudublin.ie,gerard.connolly@tudublin.ie.

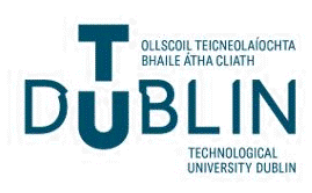




\section{Authors}

Niccolo Casnici, Marco Castellani, Flaminio Squazzoni, Manuela Testa, and Pierpaolo Dondio 


\title{
Adaptive Heuristics That (Could) Fit: Information Search and Communication Patterns in an Online Forum of Investors Under Market Uncertainty
}

Social Science Computer Review 2019, Vol. 37(6) 734-749

(C) The Author(s) 2018 Article reuse guidelines: sagepub.com/journals-permissions DOI: 10.1 |77/08944393187944I2 journals.sagepub.com/home/ssc

@SAGE

\author{
Niccolò Casnici', Marco Castellani', Flaminio Squazzoni', \\ Manuela Testa', and Pierpaolo Dondio
}

\begin{abstract}
This article examines information-search heuristics and communication patterns in an online forum of investors during a period of market uncertainty. Global connections, real-time communication, and technological sophistication have created an unpredictable market environment. As such, investors try to deal with semantic, strategic, and operational uncertainty by following heuristics that reduce information redundancy. In this study, we have tried to find traces of cognitive communication heuristics in a large-scale data set including 8 years of online posts (2004-20I2) for a forum of Italian investors. We identified various market volatility conditions on a daily basis to understand the influence of market uncertainty on cognitive and communication processes. We found that investors communicated more dynamically when the market was unstable, while they were more prone to anchor heuristic when market uncertainty was invariant. Furthermore, abnormal market trends triggered more availability-based communication patterns. We also found that expertise matters. This would suggest that online communities need intelligent, context-specific tools to support partner selection and stimulate nonredundant communication.
\end{abstract}

\section{Keywords}

financial markets, online investors, cognitive heuristics, communication, uncertainty

User-generated content and instant messaging in online social media become crucial when uncertainty grows in the real world. This is true during periods of political instability (Evans, Brown, \& Wimberly, 2018; Lotan et al., 2011; Theocharis, Lowe, van Deth, \& García-Albacete, 2015), natural disasters (Qu, Huang, Zhang, \& Zhang, 2011; Sreenivasan, Lee, \& Goh, 2011; Vieweg, Hughes, Starbird, \& Palen, 2010), epidemic emergencies (Fung et al., 2015; Hagen, Keller, Neely, DePaula,

\footnotetext{
' University of Brescia, Brescia, Italy

${ }^{2}$ Dublin Institute of Technology, Dublin, Republic of Ireland

Corresponding Author:

Flaminio Squazzoni, University of Brescia, Via San Faustino 74/B, 25 I 22 Brescia, Italy.

Email: flaminio.squazzoni@unibs.it
} 
\& Robert-Cooperman, 2017; Paul, Dredze, \& Broniatowski, 2014), and market volatility fluctuations (Casnici, Dondio, Casarin, \& Squazzoni, 2015; Piñeiro-Chousa, Vizcaíno-González, \& PérezPico, 2017; Sprenger, Tumasjan, Sandner, \& Welpe, 2014).

In finance, real-time communication on a global scale has made rational expectations of investors strongly dependent on information signals and communication (Squazzoni, 2013). In order to predict market trends, investors extract relevant information from multiple sources, including peer-to-peer platforms, whose signals require intelligent interpretation. This determines an unpredictable environment in which investors try to reduce semantic, strategic, and operational uncertainty by processing a huge amount of information, much of which is often redundant (Beunza \& Stark, 2012; Casarin \& Squazzoni, 2013).

Recent research on financial markets found that investors are often driven by cognitive heuristics. Due to subjective elements of decision-making, investors tend to follow heuristics and emotional shortcuts especially when under time and resource constraints (Monti, Boero, Berg, Gigerenzer, \& Martignon, 2012; Saavedra, Duch, \& Uzzi, 2011). While standard accounts of rational decisionmaking would conceive these as cognitive biases, studies on adaptive rationality that consider the link between decisions and environment found that investors often follow simple heuristics, as these help them cope with market uncertainty and unpredictability (Gigerenzer, 2008).

This condition, however, does not only apply to information search. Today, investors communicate directly and are engaged in collective cognitive processes of market interpretation, which leave visible traces in online posts, comments, or tweets (Casnici et al., 2015). Investigating communication patterns between investors mediated by online peer-to-peer platforms could help us understand the impact of uncertainty and unpredictability on the cognitive processes of market interpretation. This requires integrating social cognition and behavioral analysis in order to examine-although only through an indirect process of evaluation - certain cognitive mechanisms that could explain collective patterns (Lambert \& Sherer, 2013).

Our article aims to provide an example of this integration. We analyzed a large-scale data set of messages that a large group of investors exchanged in the forum website "finanzaonline.com." We attempted to reconstruct anchoring and availability effects on information search and communication (Tversky \& Kahneman, 1973). Our aim was to verify any possible context-specific links between decisions and market environments. It is reasonable to suppose that during times of low market uncertainty, investors are less prone to explore and search for information as they communicate preferentially with investors with whom they may never have interacted before. On the other hand, high uncertainty could increase stress and panic so that investors may tend to look for safety and certainty by remaining anchored to investors with whom they have already communicated with. However, in order to face higher uncertainty, investors could be strategically induced to communicate with more active investors (availability effect).

Examining these mechanisms could help us consider important aspects of these two heuristics in finance (Campbell \& Sharpe, 2009; Kaustia, Alho, \& Puttonen, 2008; Kliger \& Kudryavtsev, 2010). Improving our understanding of the link between market uncertainty and peer-to-peer communication between investors could also help contemplate certain options to promote communication in online communities (Booth, 2012; Jacobsen, 2015).

\section{Background}

The fact that financial markets depend on behavioral and social factors, such as culture and cognitive frames, was first suggested by certain pioneering studies in sociology, which considered social relationships as external frames for market exchanges (Granovetter, 1985; White, 1981). For instance, Krippner (2002) suggested placing social relationships and trust at the core of market foundations. More recently, Preda (2007) emphasized the importance of existing relationships as 
interpretative devices that create knowledge on price, quality, and reliability (Preda, 2007), while Esposito (2013a) argued that investors' interpretations are reflexive and recursive as if markets were "beauty games" with a performative nature.

Research shows that in order to cope with market uncertainty and turbulence, investors try to anticipate market fluctuations by means of mutual (direct or indirect) reflexive observations (Casnici et al., 2015; Squazzoni, 2013). Under such reflexive conditions, the usual toolbox of "rational," computational decision techniques may be (at least) ineffective (Davis, 2013), whereas a heterogeneous set of heuristics, instinctive reactions, and decision-making shortcuts may be more appropriate.

These common behavioral heuristics, or put simply, rules of thumb of human decision-making, include availability and anchoring heuristics. Tversky and Kahneman (1973, p. 208) suggested that "a person is said to employ the availability heuristic whenever he estimates frequency or probability by the ease with which instances or associations can be brought to mind." They also found that individuals often make estimations by establishing an initial value (an anchor) possibly arising from a selective attention process on the task, or possibly a partial computation. They then adjust this initial value upward or downward to make their decision. Ashton and Ashton (1988) noted that "anchoring and adjustment" heuristics are frequently used for probability assessment.

Gigerenzer and Brighton (2009) agreed with Kahneman on the centrality of heuristics in decision-making, although he also pointed out that heuristics are not cognitive mistakes or limits of rationality. In some cases, they are simply a more effective way to make a choice (Gigerenzer, 1996). He also suggested that viewing decisions as a context-free construct can limit our understanding as to why context and cognition interact with each other. In his opinion, fast and frugal reasoning is part of a toolbox of solutions and contexts that coevolved over time (see the concept of "ecological rationality" in Todd, 2001).

On the one hand, Gigerenzer (2001) showed that many financial decisions are driven by bounded rationality and unconscious stimuli, that is, conditioned responses addressed by cultural and social norms. On the other hand, recent empirical studies have found that boundedly rational investors who share information through web platforms can collectively generate relevant knowledge, which is in turn eventually incorporated into market dynamics (e.g., prices and stock returns; Bollen, Mao, \& Zeng, 2011; Casarin \& Squazzoni, 2013).

Understanding coevolution between investors' beliefs and social contexts of communication requires first to consider that the environment in which investors live today resembles the socalled ill-structured conditions originally considered by Simon (1973). Indeed, investors deal with uncertainty either by filtering social information or by contributing to its development via decentralized, direct communication (see here the concept of "docility" in Simon, 1973, 1993; Tolman, 1948).

An example of this is Casnici, Dondio, Casarin, and Squazzoni (2015) who found that the investors' communication networks react to market trends in different ways. While less turbulent market phases imply less communication, higher market volatility generates more complex communication patterns. The information content of messages is less technical in uncertainty conditions, while bad news has a different impact on network behavior, depending on market uncertainty.

Racca, Casarin, Squazzoni, and Dondio (2016) examined the impact of market uncertainty shocks on an investors' online forum by measuring knowledge dynamics and investor behavior before and during the recent financial crisis. They found that market uncertainty had a dramatic effect on investor population dynamics and on other shared knowledge, mainly due to crisis evolution. While less expert investor behavior changed during the evolution of a crisis, by becoming more sensitive to external shocks, expert investors typically considered their response to shocks both before and during the crisis. 
These studies indicate that cognitive processes and social processes of communication are constructively co-linked with financial market behavior, especially when uncertainty prevails and price interpretation is key to investors' survival. However, it is difficult to disentangle specific cognitive processes that could explain the emergence of communication patterns and their relationship with market prices and trends. Our research aims to advance our understanding of this cognitive, social, and economic "nexus."

\section{Method}

\section{Data Collection}

We analyzed 8 years of messages exchanged between investors on the forum website "finanzaonline.com" (2004-2012). The financial community of this website is the largest in the Italian Market, and the forum has a total of 162,417 members, 782,011 threads, and 28,090,299 messages. For each message, we obtained title thread, thread ID, message ID, user ID (alphanumeric code used for a privacy reasons), title, date and time of dispatch and full text, and whether the message cited (replied to) another message.

It is important to note that the forums had an asynchronous communication structure, both longitudinal and sequential, in that investors could not communicate simultaneously (as typically occurs in a chat room), but rather shared messages in chronological order and with no time limits. In the discussion under each message, there was a command "quote" that allowed anyone to intervene and comment directly on any specific message.

These forums attracted different types of investors, from newcomers who simply logged on to ask experts who had already accumulated experience in trading and were specialized in a particular title (Casarin, Casnici, Dondio, \& Squazzoni, 2015).

The forum was divided into the following subcategories: operational finance threads, finance insights, live meeting and free discussion, assistance, and archives. We focused on three topics only (i.e., "rooms"): Italian Market (FTSEMIB), Italian Small Cap, and Forex, as they had the largest number of messages and were more focused on markets and investments, which are more relevant for Italian investors. Preliminary qualitative analysis (face-to-face interviews with investors and finanzaonline.com managers) showed that these three rooms were joined by different sets of investors: Italian Market included both novices and experts, while in the other two (Small Cap and Forex) experts were predominant.

Figure 1 shows a snapshot of the homepage of the forum. Rows indicate discussion rooms, within which discussions are divided in specific topics, which are called "threads" (see Figure 2). These concern single stocks, trading techniques, market information, or any relevant event that investors think having certain financial implications. Figure 3 shows an example of the asynchronous communication structure of the forum.

\section{Anchoring}

According to the anchor effect, people are said to be anchored when they refer to a construct, belief, or opinion from which they cannot deviate because of the risk of uncertainty. To reconstruct this, we analyzed citations of each subject and measured whether they were addressed to the same investors of previous days, to never-previously quoted investors, or to the whole forum (i.e., without quoting any investor in particular).

This typically occurs when investors have sequential exchange of opinion by quoting and requoting previous information, which is often used to reassure about a belief regarding, for instance, the expected value of a stock or certain complex market trends or news. Uncertainty about the most 
Discussioni di Finanza operativa

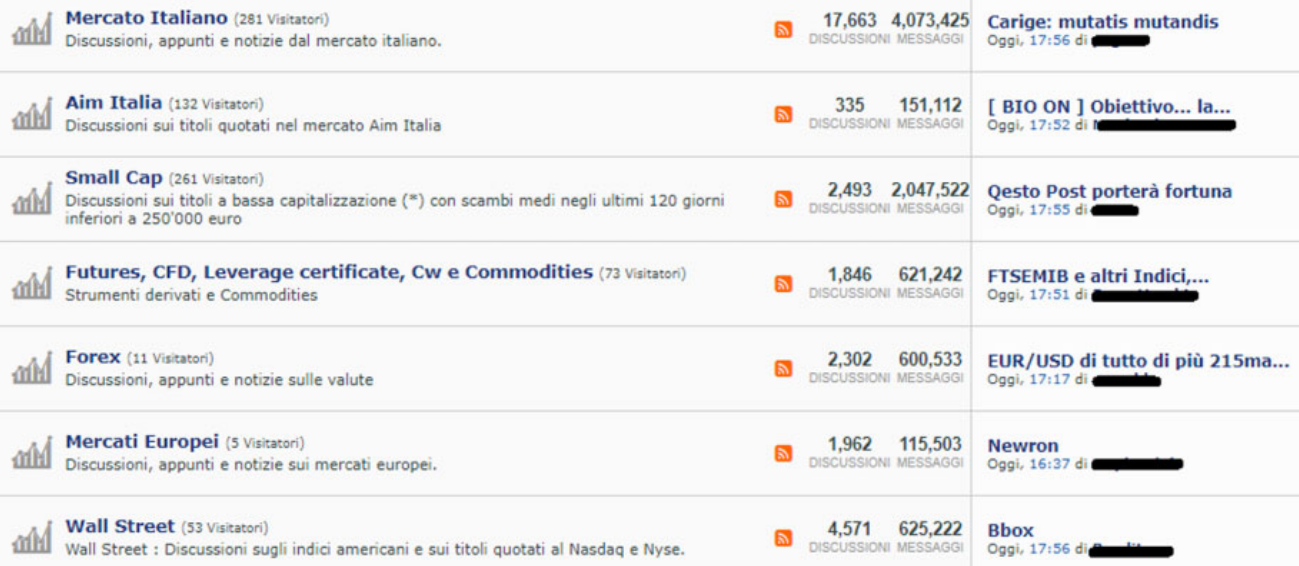

Approfondimenti di Finanza

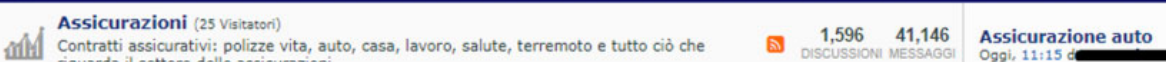
riguarda il settore delle assicurazioni

ETF, Fondi e Gestioni e Investment Certificates (78 Visitator)

ETF, Fondi e Gestioni e Investment Certificates: le vostre domande e le vostre opinioni.

\begin{tabular}{cc|c}
20,223 & 496,453 & Certificati da seguire (vol..... \\
Oggi, 17:55 d
\end{tabular}

Figure I. A snapshot of the homepage of the forum (source: finanzaonline.com).

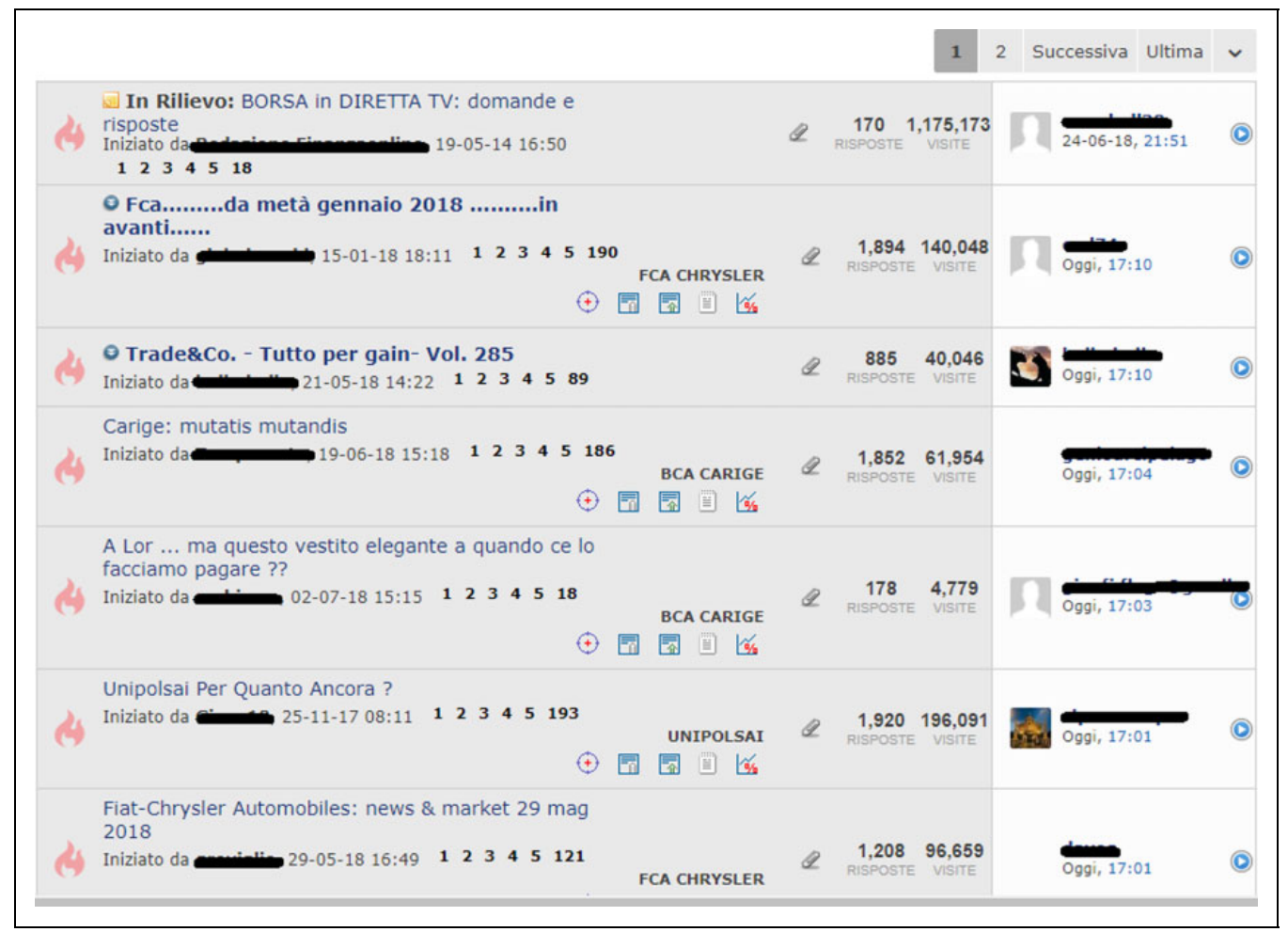

Figure 2. The most popular forum threads in 2018 (source: finanzaonline.com). 


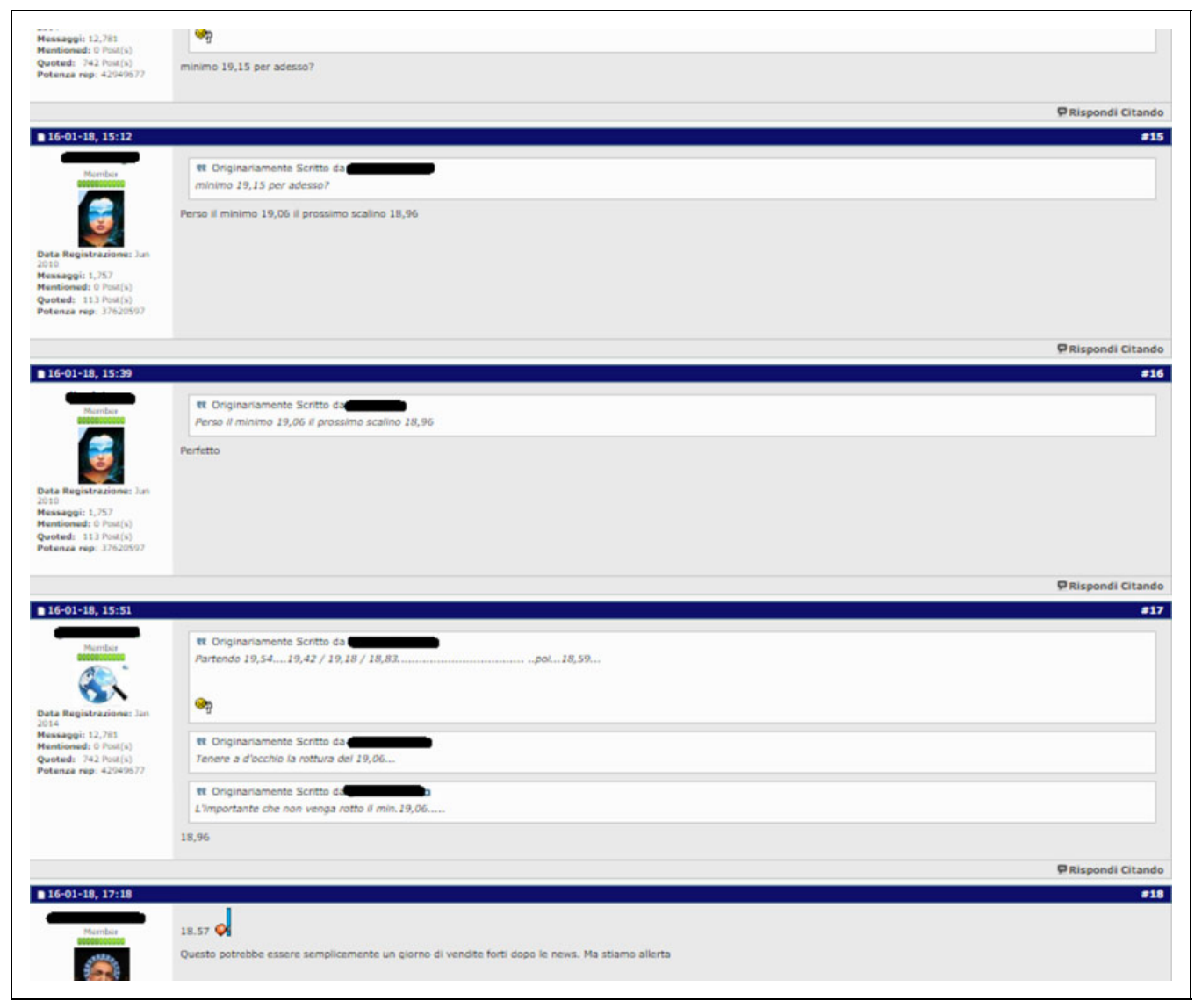

Figure 3. An example of the communication structure of the forum (source: finanzaonline.com).

appropriate interpretation is solved by relying on other users whose opinion has been previously considered (Ashton \& Ashton, 1988; Casnici et al., 2015).

Figure 4 shows the distribution of citations by a selected user (\#31654). This user was involved, for instance, in a sequence of preferential communication with 13 users in 2 years, from January 2006 to December 2007. In this period, he or she communicated with other users very frequently in some subsequent days. For example, he communicated with User 1 on January 25, 2006, 11:09 a.m. $(\# 31654 \rightarrow \# 1), 11: 10$ a.m. (\#31654 \#1), 11:12 a.m. (1 $\rightarrow \# 31654), 12: 29$ p.m. (\#1 $\leftarrow \# 31654)$, and 12:39 p.m. (\#31654 $\leftarrow \# 1$ ); and on January 26, 2006, 12:44 p.m. (\#31654 $\rightarrow \# 1$ ), 12:55 p.m. (\#31654 $\leftarrow \# 1$ ), and 1:25 p.m. (\#31654 $\rightarrow \# 1$ ). While these sequences of communication with preferential users can help to develop common understanding of market events and news, they could also lead investors to reduce information search (Campbell \& Sharpe, 2009; Casnici et al., 2015).

In our analysis, we examined the posting activity of online forum users from the Italian Market, Small Cap, and Forex rooms.

For each investor $i$ and for each day $t$ in which the investor was active on the forum, we defined the set $Q_{t_{1}}$ including all investors quoted by $i$ at Day $t_{1}$. Note that $Q_{t_{1}}$ could be an empty set, if the user wrote messages but did not quote anybody. We also considered the set $Q_{t_{2}}$ of investors quoted by $i$ at Day $t_{2}$, where $t_{2}$ represents the first day of activity of user $i$ after $t_{1}$. In general, $t_{1}$ and $t_{2}$ were subsequent days, but it was possible that the gap between $t_{1}$ and $t_{2}$ was bigger than 1 day, due to investor $i$ inactivity, often coinciding with closed market days and weekends. 


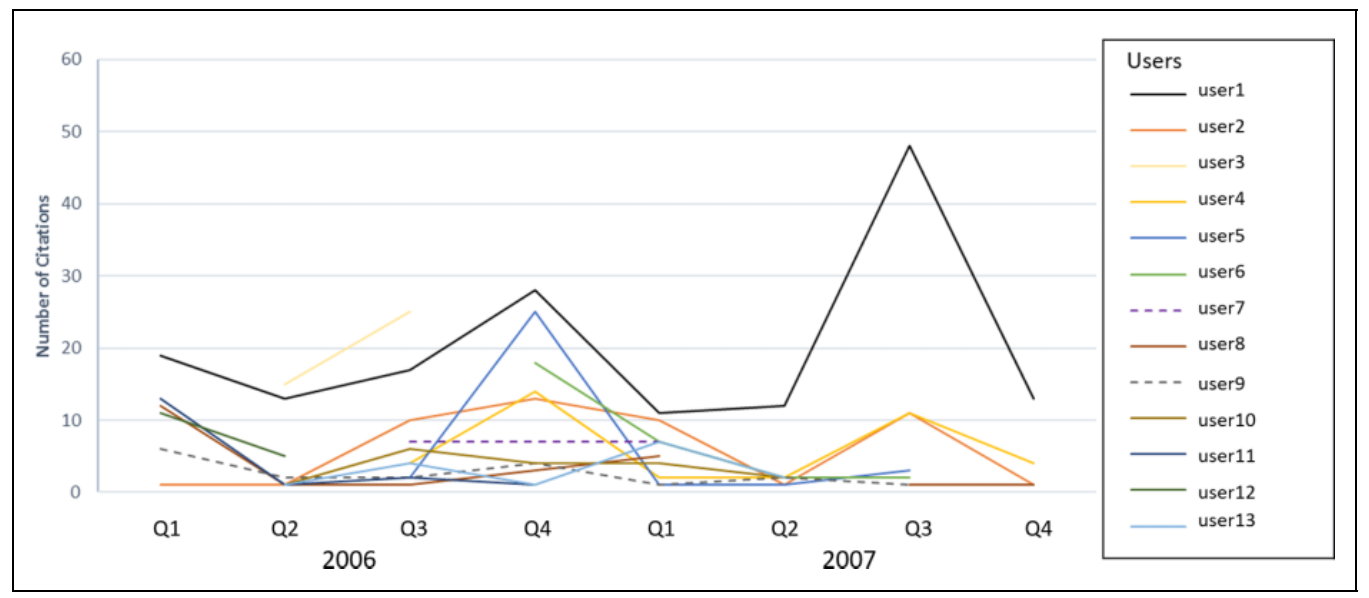

Figure 4. Distribution of citations submitted by user 31654 by cited user over time.

In order to study investors' attitude to anchoring, we quantified the difference between the sets $Q_{t_{1}}$ and $Q_{t_{2}}$, for each user and Days $t_{1}$ and $t_{2}$. We used the Jaccard index to identify investors quoting the same set of investors during subsequent days. The Jaccard index is a measure of the similarity between two sets A and B and is defined as the cardinality of the intersection divided by the cardinality of the union of the two sets:

$$
J(A, B)=\frac{|A \cap B|}{|A \cup B|} .
$$

For each user, we selected only messages with interval $t_{2}-t_{1} \leq 2$. This was reasonable given that a possible anchor could occur in a short number of days (note that 2 was also the median of the total distribution of the distances between two messages). A single record in our anchoring data set was, therefore, represented by a user_id, the Day $t_{1}$, the Day $t_{2}$, and the value of the Jaccard index quantifying the similarity between the two sets of investors quoted by the user in $t_{1}$ and $t_{2}$. We assigned a "behavior" to each record in the data set according to the following rule: If the Jaccard index associated to the record was " 0 ," this meant that at Day $t_{2}$, the user did not quote any investors that they had quoted during the previous day's activity $\left(t_{1}\right)$, alternatively, they did not quote any investor during both days. Since we considered quoting (or not quoting) as an intentional decision and consequently as a behavior pattern, we assumed that every record with an associated Jaccard index equal to " 0 " corresponded to an exploration behavior, that is, the act of looking for new information in the potential sphere of the forum. On the other hand, if the Jaccard index was equal to " 1 ," it meant that at Day $t_{2}$, an investor did write one or more messages that quoted all investors that they had quoted in the previous day's activity $t_{1}$. In this case, we assumed this behavior as anchoring, that is, the act of seeking information only from already known and recently activated sources.

Our analysis started by considering each record with Jaccard index equal to " 0 " (=no quoting at all, or not quoting recent contacts) as exploration behavior and each record with Jaccard index higher than " 0 " (=quoting at least 1 recent contact) as anchoring behavior. Subsequently, we relaxed this assumption and compared the investors' communication patterns by looking at who behaved in the most anchoring (or exploring)-oriented manner, that is, who cited more recent contacts. 


\section{Availability}

To examine availability, we selected a subsample consisting of 265,520 messages from the Italian Market, Small Cap, and Forex rooms that contained a quotation to other investors' messages. According to the availability effect, people's judgment tends to rely more heavily on their more recent and visible information, making new opinions biased toward the latest news. To reconstruct this, we looked at whether an investor $i$ posting a message at time $t_{i}$ tended to quote investors who recently wrote more messages, that is, were more visible. This required constructing an "availability ranking" of the most active users at time $t_{i}$ for each thread. We ranked users based on the number of messages written by each user in a window of the 100 most recent messages written before time $t_{i}$ in the thread (as it appeared improbable that a user would draw on older information and the forum does not allow users to quote a message belonging to another thread). We considered investors quoting highly visible users (i.e., a user with high "availability ranking") as performing availability-oriented behavior.

\section{Market Volatility}

Following Casnici et al. (2015), in order to understand the role of market uncertainty in shaping communication process, we identified high-volatility and low-volatility periods in market trends on a daily basis. We reconstructed the volatility of our 2004-2012 time series from the Bloomberg website. We used two different volatility indices as data source, one for Italian Market and Forex (FTSEMIB index) and another for Small Cap (FTSE Italia Small Cap index). Since there was no information about the index during weekends and holidays, despite investors also communicating on these days, we assigned the volatility of the previous nearest working day to those days where the index was not calculated.

We identified two different volatility conditions: volatility variations (used to study anchoring heuristic) and volatility regimes (used to study availability heuristic). In the first case, three groups of messages were formed depending on the variation between the volatility of the day in which each of the message was generated $\left(V_{t_{2}}\right)$ and the volatility of the last quoting day $\left(V_{t_{1}}\right)$. Then, volatility variations $\left(\Delta=V_{t_{2}}-V_{t_{1}}\right)$ were defined according to the following criteria: decreasing $=$ \{messages: $\left.\Delta<\mathrm{Me}^{-}\right\}$; invariant $=\left\{\right.$messages: $\left.\mathrm{Me}^{-}<\Delta<\mathrm{Me}^{+}\right\}$; increasing $=\left\{\right.$messages: $\left.\Delta>\mathrm{Me}^{+}\right\}$, where $\mathrm{Me}^{-}$was the median of negative deviations (i.e., the volatility decreases) in the whole series and $\mathrm{Me}^{+}$was the median of positive deviations (i.e., the volatility increases). We considered this indicator as a proxy of the investors' perceived market uncertainty.

Concerning availability heuristics, preliminary analysis showed that in $90 \%$ of cases, quotations occurred during the same day. Therefore, it was useless to check the variation of volatility between the postquoting and the postquoted. For this reason, we defined a second indicator (i.e., volatility regimes) as follows: Three groups of messages were formed according to the volatility of the day in which each message was generated: high ( $V>66$ th centile of the whole volatility series), medium (33rd centile $<V<66$ th centile), and low ( $V<33$ th centile). As in the previous case, we considered this indicator as a proxy of the investors' perceived market uncertainty.

\section{Results}

\section{Anchoring}

After preliminary data preparation, we obtained a data set with 383,236 rows, in which an investor interacted with other investors quoting their messages over a short time interval (maximum 2 days). Of those rows, 139,118 came from Italian Market's topic, 21,094 from Forex's topic, and 223,024 
Table I. Distribution of Behaviors Grouped by Volatility Variation in Each Room.

\begin{tabular}{lccc}
\hline & \multicolumn{2}{c}{ Behavior (\%) } & \\
\cline { 2 - 3 } Volatility Variation & Exploration & Anchoring & \\
\hline Italian Market room & & & .00 \\
Decreasing & 76.52 & 23.48 & \\
Constant & 68.64 & 31.36 & \\
Increasing & 77.13 & 22.87 & .00 \\
Small Cap room & & & \\
Decreasing & 67.62 & 32.38 & \\
Constant & 56.98 & 43.02 & .00 \\
Increasing & 67.87 & 32.13 & \\
Forex room & & & \\
Decreasing & 63.51 & 36.49 & .00 \\
Constant & 50.86 & 49.14 & \\
Increasing & 62.63 & 37.37 & \\
Decreasing & 70.88 & 29.12 & \\
All & & 39.43 & \\
Constant & 60.57 & 29.08 & \\
Increasing & 70.92 & & \\
\hline
\end{tabular}

Note. Volatility variation conditions are defined as the variation between the volatility of the day in which each message was generated $\left(V_{t_{2}}\right)$ and the volatility of the last quoting day $\left(V_{t_{1}}\right)$. Decreasing $=\left\{\right.$ messages: $\left.\Delta<M e^{-}\right\}$; invariant $=\{$messages: Me $\left.<\Delta<\mathrm{Me}^{+}\right\}$; increasing $=\left\{\right.$messages: $\left.\Delta>\mathrm{Me}^{+}\right\}$, where $\mathrm{Me}^{-}$and $\mathrm{Me}^{+}$were the median of negative and positive deviations (i.e., the volatility decreases or increases, respectively) in the whole series. Records with Jaccard index equal to " 0 " (=no quoting anybody or not quoting recent contacts) were categorized as "exploration"-oriented behaviors and records with Jaccard index higher than " 0 " (=quoting at least one recent contact) were categorized as "anchoring"-oriented behaviors.

from Small Cap's topic. There were 8,349 investors in total; 2,506 were involved in the Italian Market's topic, 1,075 in the Forex's topic, and 5,587 in the Small Cap's topic.

Table 1 shows the percentage of investors who behaved differently according to variations in volatility. In each room, exploration was more frequent when volatility was abnormal (i.e., increasing and decreasing) and conversely, anchoring was more frequent when volatility was invariant. Pearson $\chi^{2}$ test indicated that behavior and volatility conditions were significantly associated in all three rooms.

In order to further explore the interplay between behavior and market uncertainty, we looked at Jaccard index values under different volatility conditions in more detail. We performed a one-way analysis of variance to determine whether the Jaccard index was statistically different when volatility changed, for the whole sample and for each room (see Table 2). Concerning the whole data set, the Bonferroni post hoc test confirmed that the Jaccard index was significantly higher in the constant compared to decreasing volatility periods $(p=.00)$ and was significantly lower in increasing volatility compared to constant volatility periods $(p=.00)$. We found no statistically significant differences between increasing and decreasing periods $(p=.112)$. Furthermore, we performed the same test for each of the three rooms separately. The results were perfectly consistent with the general trend (see Table 2).

Our results indicate that while conditions of market uncertainty (i.e., abnormally low or high volatility) triggered an explorative-oriented behavior, low market uncertainty was associated with conservative in-depth communication styles. This was true for all forum rooms. According to Casnici et al. (2015), investors preferably communicate with already known investors when market uncertainty is lower. On the contrary, in situations of high volatility, investors need to revise expectations quickly and tend to build more unstable patterns of communication. 
Table 2. Summary of Jaccard Similarity: Whole Sample and Single Rooms.

\begin{tabular}{lcccc}
\hline Volatility Variation & Mean & Standard Deviation & Frequency & $F$ Test \\
\hline Italian Market room & & & & \\
$\quad$ Decreasing & .031 & .086 & 50,429 & \\
Constant & .038 & .088 & 42,547 & $F(2,|39| 15)=128.46, p=.001$ \\
$\quad$ Increasing & .030 & .086 & 46,142 & \\
Small Cap room & & & & \\
$\quad$ Decreasing & .086 & .178 & 69,404 & \\
Constant & .104 & .176 & 79,392 & $F(2,223021)=262.22, p=.00$ \\
Increasing & .086 & .178 & 74,228 & \\
Forex room & & & & \\
Decreasing & .102 & .194 & $7,84 \mid$ & \\
Constant & .122 & .188 & 6,018 & $F(2,21091)=22.05, p=.00$ \\
Increasing & .104 & .198 & 7,235 & \\
All & & .153 & 127,674 & \\
Decreasing & .065 & .156 & 127,957 & $F(2,383233)=519.9, p=.00$ \\
Constant & .083 & .156 & 127,605 & \\
Increasing & .066 & &
\end{tabular}

Note. Volatility variation conditions are defined as the variation between the volatility of the day in which each message was generated $\left(V_{t_{2}}\right)$ and the volatility of the last quoting day $\left(V_{t_{1}}\right)$. Decreasing $=\left\{\right.$ messages: $\left.\Delta<\mathrm{Me}^{-}\right\}$; invariant $=\{$messages: Me $\left.<\Delta<\mathrm{Me}^{+}\right\}$; increasing $=\left\{\right.$messages: $\left.\Delta>\mathrm{Me}^{+}\right\}$, where $\mathrm{Me}^{-}$and $\mathrm{Me}^{+}$were the median of negative and positive deviations (i.e., the volatility decreases or increases, respectively) in the whole series.

Our findings indicate that less experienced investors writing in the Italian Market room tended to be less prone to the anchoring effect, compared to other rooms. Here, novice investors showed different information-seeking strategies: A lack of experience in the field may have been associated with weaker ties with other investors, which could have triggered less structured interactions and more explorative-based behavior.

\section{Availability}

In order to examine availability, we used a data set of 265,520 rows, which indicated every post with a quote. Six thousand seven hundred and eighty posts were written in Forex room, 142,879 in Italian Market room, and 115,861 in Small Cap room.

The results showed that availability heuristic was preponderant, even without considering market volatility. Although the variable rank of all investors ranged between 1 and 88, the median of quoted investors was 4, while the mean was 6.26. Moreover, we analyzed availability heuristics under different market volatility conditions. Our analysis confirmed that volatility regimes were significantly associated with the rank of quoted investors for the whole sample (see Table 3 ). The Bonferroni post hoc test showed that the rank of quoted investors was higher in the low regime compared to the medium regime $(p=.00)$, but higher in the high regime compared to the medium one $(p=.00)$. However, there were no statistically significant differences between high- and lowvolatility regimes.

We ran the same test independently on the three rooms. In general, we found the same trend in all rooms, with medium-volatility regimes associated with the lowest average rank of quoted investors. This meant that all investors, independent of rooms, topics, or expertise, tended to be more availability-oriented and use information-seeking strategies under normal market conditions. However, investors' behavior under abnormal market conditions was influenced by context-related variables. Indeed, we found that in the Italian Market room, the average ranking of quoted investors 
Table 3. Summary of Rank of Quoted Investors: Whole Sample and Single Rooms.

\begin{tabular}{lcccc}
\hline Volatility Regime & Mean & Standard Deviation & Frequency & $F$ Test \\
\hline Italian Market room & & & & \\
$\quad$ Low & 5.914 & 5.52253 & 47,592 & \\
$\quad$ Medium & 5.846 & $5.405 I I$ & 47,517 & $F(2$, II 5858) $=39.15, p=.00$ \\
$\quad$ High & 6.086 & 5.80033 & 47,770 & \\
Small Cap room & & & & \\
$\quad$ Low & 6.825 & 6.71969 & 38,430 & \\
$\quad$ Medium & 6.413 & 6.33979 & 38,875 & $F(2,142876)=23.43, p=.00$ \\
$\quad$ High & 6.667 & 6.54349 & 38,556 & \\
Forex room & & & & \\
$\quad$ Low & 6.865 & 6.92706 & 2,251 & \\
$\quad$ Medium & 5.824 & 5.81253 & 2,264 & $F(2,6777)=14.87, p=.00$ \\
$\quad$ High & 6.464 & 6.63809 & 2,265 & \\
All & & & & \\
Low & 6.335 & 6.126 & 88,273 & \\
$\quad$ Medium & 6.094 & 5.85 & 88,656 & $F(2,265517)=49.66, p=.00$ \\
High & 6.349 & 6.163 & 88,591 & \\
\hline
\end{tabular}

Note. The volatility for each daily observation $i$ was categorized as "high regime" if $V_{i}>66$ th centile of the whole volatility series, "medium regime" if $33 \mathrm{rd}$ centile $<V_{i}<66$ th centile, and "low regime" if $V_{i}<33$ th centile.

was significantly lower in the low and medium regimes compared to the high regime $(p=.00)$. Ranking under low-volatility regime was not significantly different from ranking under mediumvolatility regimes.

In the case of the Small Cap room, the low regime had a higher ranking than both medium and high regimes ( $p=.00$ and $p=.003$, respectively). The high regime being significantly higher than the medium regime $(p=.00)$. Finally, our findings showed that Italian Market investors tended to be less inclined to availability heuristics than those in the other two rooms. This occurred independent of uncertainty conditions. Indeed, when market volatility was low, the average quote ranking of Italian Market investors was lower than that of Small Cap and Forex investors. The same occurred in case of medium and high volatility, except the case of Italian Market and Forex in medium volatility.

\section{Discussion and Conclusion}

This article aimed to examine cognitive mechanisms of information search and communication followed by online investors under various market conditions. By using a large-scale data set of online investors of the Finanzaonline.com community in Italy, we focused on two key decisionmaking heuristics, that is, anchoring and availability, which we expected may be involved differently under periods of market uncertainty (Campbell \& Share, 2009; Casnici et al., 2015).

While anchoring and availability effects are often considered in theory as "deviations" from full rationality, real investors may use them to adapt intelligently to different market scenarios, where uncertainty must be interpreted. For instance, when market volatility is high and uncertainty prevails, investors may try to communicate with other investors with whom they were already in contact with. The opposite could also be an option: When the market is less volatile, investors may be less needy of new market interpretations and so more prone to communicate with already known contacts. The first case would in principle testify to certain cognitive limitations of investors. That is, when uncertainty prevails, any new information presumably shared by other investors while 
communicating with unrelated others could be relevant, whereas confronting interpretations with already known investors may be less relevant.

Our findings do not confirm the traditional view of heuristics as a cognitive bias (Mousavi, Gigerenzer, \& Keirandish, 2016). Furthermore, our results indicate that online investors adapted their behavior to different scenarios, as abnormal market conditions (low or high volatility) were associated with different information-seeking strategies. Indeed, when uncertainty increased, investors were less prone to the anchoring effect and tended to explore other possible contacts, which were mainly selected by following availability-based criteria.

According to Casnici et al. (2015), investors preferably communicated with already known investors when market uncertainty was perceived as normal, because either they were searching for in-depth trusted information or trying to find confirmation of their own beliefs. In the opposite case, under situations of volatility variation (increasing or decreasing) and external shocks, investors needed to revise expectations quickly and so look at different sources, which determined more unstable patterns of communication. If we consider periods of constant volatility as circumstances in which financial markets were less risky and did not challenge investors' perceptions, following anchoring heuristics - that is, communicating preferably with the same subjects - was fully appropriate. When volatility tended to vary, investors perceived such uncertainty by exploring new contacts and seeking new market interpretations.

Market uncertainty is also associated with availability effects. Investors tended to react to abnormal volatility (high or low) by seeking more immediately available information, that is, quoting highly active and visible users. Being exposed to ambiguous signals and facing unexpected market events, investors needed to elaborate quick strategies. In the absence of trustworthiness indicators of other investors, they probably considered the amount of messages posted as being positively related to personal expertise and tended to communicate with more active and available users. However, significant differences were found between regimes among rooms. On the one hand, in Small Cap and Forex, availability prevailed especially during low volatility. On the other hand, in the Italian Market room, availability was followed more when market volatility was high.

Our findings also showed intraroom differences. Investors from the Italian Market room, who were less experienced than others, were less inclined both to anchoring and availability heuristics, and communicated by following less structured patterns. This would confirm that investors probably perceived the link between heuristics and contexts differently depending on their experience, thus confirming the role of expertise in interpreting the market and relying more intelligently on online communication (Casarin et al., 2015). This shows that heuristics and communication between investors are context-specific processes (Casnici et al., 2015; Squazzoni, 2013).

With all caveats due to the case-based nature of our study, our findings suggest the need for expanding the concept of "rationality" toward the idea of distributed, socially embedded cognition, where cognition is mediated by social communication (Secchi, 2011; Secchi \& Cowley, 2018; Weick \& Sutcliffe, 2006). Here, it is likely that online technologies enable this distributed effort of market interpretation by constraining attention, organizing peer-to-peer communication, and stimulating mutual reference in sense-making processes, while at the same time increasing subjective uncertainty and the need for adaptive heuristics (Monti et al., 2012; Squazzoni, 2013).

The context-specific nature of the heuristics of partner selection and communication and certain differences between experts and novices suggest interesting managerial and technological implications. If we consider that website forums are often not supported by advanced search and communication tools, rarely customized to the needs of users with different expertise (Casnici et al., 2015), implementing options for partner selection and communication by modifying content access, mapping relevant opinion makers, and stimulating content sharing could help (Booth, 2012; Jacobsen, 2015). 
In this respect, recent implementations of reputation and recommendation systems suggest that not only do collaborative filtering algorithms, rankings, and other personalization tools help coordination, communication, and information search; they also increase participation and knowledge development in online communities (Adamopoulos \& Tuzhilin, 2013; Malinen, 2015). However, the challenge in financial online communities is developing tools that can adapt to varying market conditions as critical events and volatility have serious implications on communication patterns (Casnici et al. 2015). Indeed, while in certain online communities, the flux of communication between users can have picks but activities are less dependent on exogenous factors, content sharing and communication in financial communities could strongly depend on market volatility, with disproportional impact of certain exogenous events (Casarin \& Squazzoni, 2013; Casnici et al., 2015).

Here, our findings suggest that contexts truly matter. What investors need in periods of relative market uncertainty may not be what they need in periods of high uncertainty. Customizing online tools on user characteristics without considering the changing nature of the environment in which they live may lead to unintended effects.

This finding has a more general implication. Our results indicate that considering heuristics as cognitive limitations or merely deviations from "full rationality" could be misleading especially in contexts in which information is not scarce and uncertainty prevails (Kirman, 2014). In these cases, Gigerenzer's idea of ecological rationality as a set of heuristics and tools that are context dependent could be the key. The efficacy of certain rules and behavior could reflect a context-specific evolutionary selection, through trial and error experiments performed by many individuals. Complex sociocognitive scaffolds, such as financial markets, are probably one of these contexts and this is why they are attracting a lot of attention (Todd \& Gigerenzer, 2012). In this area, more research on the interplay of cognition and social processes of communication and market behavior is needed to understand the specific distributed nature of the aggregate intelligence of markets in complex environments (Bertolotti \& Magnani, 2017).

Obviously, our study has certain limitations. First, while our analysis captures certain aggregate patterns, we did not look at the content of communications. Examining how topics on which investors communicated changed during different volatility regimes could reveal interesting insights on the self-reinforcing nature of contexts, communication, and meaning (Cowley \& ValléeTourangeau, 2017).

Second, our data did not include investment decisions by forum users. Examining the interplay of peer-to-peer communication and investment decisions would be crucial to shed light on the complex reflexive nature of modern financial markets (Casnici et al., 2015; Dow, 2010). This could also expand the conventional approach to macro-micro feedback in finance (e.g., Bond, Edmans, \& Goldstein, 2012) toward including more complex meso (peer-to-peer communication) processes (Esposito, 2013a, 2013b).

Furthermore, our study did not consider the structure of communication over time. This could help to reveal the emergence of endogenous constraints within the forum, in specific rooms or threads. For instance, representing user interactions as advice networks could improve our understanding of microfoundations of collective, distributed cognition and help us discover means to improve the efficiency of recommendation and reputation systems in financial platforms.

Finally, although we found aggregate traces of cognitive heuristics of information search and communication, only in-depth analysis at the individual and interpersonal level could reveal the emerging sociocognitive patterns of second-order "observation" in detail (Esposito, 2013a; Stark, 2013). In general, we could only show that cognitive aspects of information search and communication could be inferred even from a large-scale, aggregate, and complex data set. 


\section{Authors' Note}

For any information about the database used in this study and access to metadata for replication, please contact the corresponding author at flaminio.squazzoni@unibs.it. The authors would like to thank Antonio Pugliese (Browne Editore) for sharing finanzaonline.com data, Luca Brognara for information on the forum, and Claudio Gandelli for help on the first version of the database. Usual caveats apply.

\section{Declaration of Conflicting Interests}

The authors declared no potential conflicts of interest with respect to the research, authorship, and/or publication of this article.

\section{Funding}

The authors received no financial support for the research, authorship, and/or publication of this article.

\section{References}

Adamopoulos, P., \& Tuzhilin, A. (2013). On unexpectedness in recommender systems: Or how to better expect the unexpected. ACM Transactions on Intelligent Systems and Technology, 54, 1-32.

Ashton, A. H., \& Ashton, R. H. (1988). Sequential belief revision in auditing. Accounting Review, 63, 623-641.

Bertolotti, T., \& Magnani, L. (2017). Contemporary finance as a critical cognitive niche: An epistemological outlook on the uncertain effects of contrasting uncertainty. In E. Ippoliti \& P. Chen (Eds.), Methods and finance (pp. 129-150). Cham, Switzerland: Springer International.

Beunza, D., \& Stark, D. (2012). From dissonance to resonance: Cognitive interdependence in quantitative finance. Economy and Society, 41, 383-417.

Bollen, J., Mao, H., \& Zeng, X. (2011). Twitter mood predicts the stock market. Journal of Computational Science, 2, 1-8.

Bond, P., Edmans, A., \& Goldstein, I. (2012). The real effects of financial markets. Annual Review of Financial Economics, 4, 339-360.

Booth, S. E. (2012). Cultivating knowledge sharing and trust in online communities for educators. Journal of Educational Computing Research, 47, 1-31.

Campbell, S. D., \& Sharpe, S. A. (2009). Anchoring bias in consensus forecasts and its effect on market prices. Journal of Financial and Quantitative Analysis, 44, 369-390.

Casarin, R., Casnici, N., Dondio, P., \& Squazzoni, F. (2015). Back to basics! The educational gap of online investors and the conundrum of virtual communities. Journal of Financial Management, Markets and Institutions, 3, 51-69.

Casarin, R., \& Squazzoni, F. (2013). Being on the field when the game is still under way. The financial press and stock markets in times of crisis. PLoS One, 8, e67721.

Casnici, N., Dondio, P., Casarin, R., \& Squazzoni, F. (2015). Decrypting financial markets through e-joint attention efforts: On-line adaptive networks of investors in periods of market uncertainty. PLoS One, 10, $\mathrm{e} 0133712$.

Cowley, S. J., \& Vallée-Tourangeau, F. (2017). Thinking, values and meaning in changing cognitive ecologies. In S. Cowley \& F. Vallée-Tourangeau (Eds.), Cognition beyond the brain (pp. 1-17). Cham, Switzerland: Springer International.

Davis, J. B. (2013). Soros's reflexivity concept in a complex world: Cauchy distributions, rational expectations, and rational addiction. Journal of Economic Methodology, 20, 368-376.

Dow, S. C. (2010). Cognition, market sentiment and financial instability. Cambridge Journal of Economics, 35, 233-249.

Esposito, E. (2013a). Economic circularities and second-order observation: The reality of ratings. Sociologica, $7,1-10$.

Esposito, E. (2013b). The structures of uncertainty: Performativity and unpredictability in economic operations. Economy \& Society, 42, 102-129. 
Evans, K., Brown, K. J., \& Wimberly, T. (2018). "Delete your account" the 2016 presidential race on twitter heather. Social Science Computer Review, 36, 500-508.

Fung, I. C., Hao, Y., Cai, J., Ying, Y., Schaible, B. J., Yu, C. M., .. Fu, K. W. (2015). Chinese social media reaction to information about 42 notifiable infectious diseases. PLos One, 10, e0126092.

Gigerenzer, G. (1996). On narrow norms and vague heuristics: A reply to Kahneman and Tversky. Psychological Review, 103, 592-596.

Gigerenzer, G. (2001) The adaptive toolbox. In G. Gigerenzer \& R. Selten (Eds.), Bounded rationality-The adaptive toolbox. Cambridge: The MIT Press.

Gigerenzer, G. (2008). Rationality for mortals: How people cope with uncertainty. Oxford, England: Oxford University Press.

Gigerenzer, G., \& Brighton, H. (2009). Homo heuristics: Why biased minds make better inferences. Topics in Cognitive Science, 1, 107-143.

Granovetter, M. (1985). Economic action and social structure: The problem of embeddedness. American Journal of Sociology, 91, 481-510.

Hagen, T. L., Keller, T., Neely, S., DePaula, N., \& Robert-Cooperman, C. (2017). Crisis communications in the age of social media: A network analysis of Zika-related tweets. Social Science Computer Review.

Jacobsen, G. D. (2015). Consumers, experts, and online product evaluations: Evidence from the brewing industry. Journal of Public Economics, 126, 114-123.

Kaustia, M., Alho, E., \& Puttonen, V. (2008). How much does expertise reduce behavioral biases? The case of anchoring effects in stock return estimates. Financial Management, 37, 391-412.

Kirman, A. (2014). Is it rational to have rational expectations? Mind \& Society, 13, 29-48.

Kliger, D., \& Kudryavtsev, A. (2010). The availability heuristic and investors' reaction to company-specific events. The Journal of Behavioral Finance, 11, 50-65.

Krippner, G. R. (2002). The elusive market: Embeddedness and the paradigm of economic sociology. In D. Carlston (Ed.), Theory and Society, 30, 775-810.

Lambert, A. J., \& Scherer, L. (2013). Measurement and methodology in social cognition: A historical perspective. The Oxford Handbook of Social Cognition (pp. 33-52). Oxford, New York: Oxford University Press.

Lotan, G., Graeff, E., Ananny, M., Gaffney, D., Pearce, I., \& Boyd, D. (2011). The Arab Spring. The revolutions were tweeted: Information flows during the 2011 Tunisian and Egyptian revolutions. International Journal of Communication, 5, 1375-1405.

Malinen, S. (2015). Understanding user participation in online communities: A systematic literature review of empirical studies. Computers in Human Behavior, 46, 228-238.

Monti, M., Boero, R., Berg, N., Gigerenzer, G., \& Martignon, L. (2012). How do common investors behave? Information search and portfolio choice among bank customers and university students. Mind \& Society, 11, 203-233.

Mousavi, S., Gigerenzer, G., \& Kheirandish, R. (2016). Rethinking behavioral economics through fast-andfrugal heuristics. In R. Frantz, S.-H. Chen, K. Dopfer, F. Heukelom, \& S. Mousavi (Eds.), Routledge handbook of behavioral economics (pp. 280-296). London, England: Taylor \& Francis.

Paul, M. J., Dredze, M., \& Broniatowski, D. (2014). Twitter improves influenza forecasting. PLos Currents Outbreaks, October 28, Edition 1.

Piñeiro-Chousa, J., Vizcaíno-González, M., \& Pérez-Pico, A. M. (2017), Influence of social media over the stock market. Psychology Marketing, 34, 101-108.

Preda, A. (2007). The sociological approach to financial markets. Journal of Economic Surveys, 21, 506-533.

Qu, Y., Huang, C., Zhang, P., \& Zhang, J. (2011). Microblogging after a major disaster in China: A case study of the 2010 Yushu earthquake. In Proceedings of the ACM 2011 conference on computer supported cooperative work. CSCW'11 (pp. 25-34). New York, NY: ACM.

Racca, P., Casarin, R., Squazzoni, F., \& Dondio, P. (2016). Resilience of an online financial community to market uncertainty shocks during the recent financial crisis. Journal of Computational Science, 16, 190-199. 
Saavedra, S., Duch, J., \& Uzzi, B. (2011). Tracking traders' understanding of the market using e-communication data. PLoS One, 6, e26705.

Secchi, D. (2011). Extendable rationality. Understanding decision making in organizations. New York, NY: Springer.

Secchi, D., \& Cowley, S. (2018). Modeling organizational cognition: The case of impact factor. Journal of Artificial Societies and Social Simulation, 21, 13.

Simon, H. (1973). The structure of ill structured problem. Artificial Intelligence, 4, 181-201.

Simon, H. (1993). Altruism and economics. American Economic Review, 83, 156-161.

Sprenger, T. O., Tumasjan, A., Sandner, P. G., \& Welpe, I. M. (2014), Tweets and trades: The information content of stock microblogs. European Financial Management, 20, 926-957.

Squazzoni, F. (2013). Embedded, scattered, confused minds: What do hyper-conductive markets impose on investors' social intelligence. Sociologica, 7, 16-20.

Sreenivasan, N. D., Lee, C. S., \& Goh, D. H. L. (2011) Tweet me home: Exploring information use on twitter in crisis situations. In A. A. Ozok \& P. Zaphiris (Eds.), Online communities and social computing. OCSC'2011: Vol. 6778. Lecture notes in computer science (pp. 120-129). Berlin, Heidelberg: Springer.

Stark, D. (2013). Observing finance as a network of observations. Sociologica, 7, 21-25.

Theocharis, Y., Lowe, W., van Deth, J. W., \& García-Albacete, G. (2015). Using Twitter to mobilize protest action: Online mobilization patterns and action repertoires in the Occupy Wall Street, Indignados, and Aganaktismenoi movements. Journal Information, Communication \& Society, 18, 202-210.

Todd, P. M. (2001). Fast and frugal heuristics for environmentally bounded minds. In G. Gigerenzer \& R. Selten (Eds.), Bounded rationality: The adaptive toolbox (pp. 51-70). Cambridge, MA: The MIT Press.

Todd, P. M., \& Gigerenzer, G. (2012). Ecological rationality: Intelligence in the world. Oxford, England: OUP.

Tolman, E. C. (1948). Cognitive maps in rats and men. The Psychological Review, 55, 189-208.

Tversky, A., \& Kahneman, D. (1973). Availability: A heuristic for judging frequency and probability. Cognitive Psychology, 5, 207-232.

Vieweg, S., Hughes, A. L., Starbird, K., \& Palen, L. (2010). Microblogging during two natural hazards events: What Twitter may contribute to situational awareness. In Proceedings of the SIGCHI conference on human factors in computing systems. CHI'10 (pp. 1079-1088). New York, NY: ACM.

Weick, K. E., \& Sutcliffe, K. M. (2006). Mindfulness and the quality of organizational attention. Organization Science, 17, 514-524.

White, H. C. (1981). Where do markets come from? American Journal of Sociology, 87, 517-547.

\section{Author Biographies}

Niccolò Casnici is post-doc at the Department of Economics and Management, University of Brescia, Italy. His field of research is sociology of finance and social networks.

Marco Castellani is Associate Professor at the University of Brescia, Italy, were he teaches economic sociology. Most of his research revolves around bounded rationality and decision-making.

Flaminio Squazzoni is Associate Professor at the University of Brescia, Italy, were he lead the GECS-Research Group on Experimental and Computational Sociology. He is editor of JASSS-Journal of Artificial Societies and Social Simulation and has been former president of ESSA-The European Social Simulation Association (2012-2016).

Manuela Testa has a Master Degree in Cognitive science and decision making. She attended the Ph.D. Programme in Economic Sociology and Labour Studies at the University of Brescia. Her main interest is socio-finance and evidence-based analysis of financial markets.

Pierpaolo Dondio has a PhD from Trinity College Dublin and since 2012 is Lecturer at the Dublin Institute of Technology, Ireland. His research interests are argumentation theory and online communities' behavior and dynamics. 